\title{
Hydration and Durability of Concrete Containing Supplementary Cementitious Materials
}

\author{
Xiao-Yong Wang, ${ }^{1}$ Han-Seung Lee, ${ }^{2}$ Xiao-Jian Gao, ${ }^{3}$ and Yao Luan ${ }^{4}$ \\ ${ }^{1}$ Department of Architectural Engineering, Kangwon National University, Chuncheon-si, Republic of Korea \\ ${ }^{2}$ Department of Architectural Engineering, Hanyang University, Ansan-si, Republic of Korea \\ ${ }^{3}$ School of Civil Engineering, Harbin Institute of Engineering, Harbin, China \\ ${ }^{4}$ Department of Civil Engineering, Saitama University, Saitama, Japan
}

Correspondence should be addressed to Xiao-Yong Wang; wxbrave@kangwon.ac.kr

Received 26 September 2017; Accepted 26 September 2017; Published 16 November 2017

Copyright (C) 2017 Xiao-Yong Wang et al. This is an open access article distributed under the Creative Commons Attribution License, which permits unrestricted use, distribution, and reproduction in any medium, provided the original work is properly cited.

Fly ash, slag, silica fume, and other supplementary cementitious materials (SCMs) are used more and more for producing modern concrete. SCMs can provide various benefits to the concrete industry, such as improving the workability, early-age performance, and durability of concrete structures, lowering the materials' cost of proportions of concrete mix, and reducing greenhouse gas emissions. The material performance of concrete containing SCMs is an important research theme for the sustainable development of the concrete industry.

To meet the increasing requirements about knowledge of SCMs from concrete researchers and construction companies, in 2016, we proposed this special issue. This special issue aroused the interest of researchers around the world. 26 articles were finally published after careful reviews. The acceptance rate is about $45 \%$. These articles cover different aspects of materials performance of SCMs blended concrete, such as chemical admixtures, hydration, strength development, shrinkage, chloride ingress, frost, corrosion, and service life evaluation. The integrated material-structure studies, such as the influence of load on durability and structure performance under low temperature, are also presented. In addition, the analysis of durability-induced damage and repairing of damaged concrete structures are discussed in detail. In summary, this special issue covers material scale and structure scale and considers the production stage, service stage, and repair stage of structures. The detailed experimental studies, theoretical analyses, and wide and deep discussions will contribute to the realization, utilization, and development of SCMs.

Finally, we are grateful to the authors, reviewers, and editors of this journal. The publication of this special issue embodies the efforts of those authors, reviewers, and editors.

\author{
Xiao-Yong Wang \\ Han-Seung Lee \\ Xiao-Jian Gao \\ Yao Luan
}



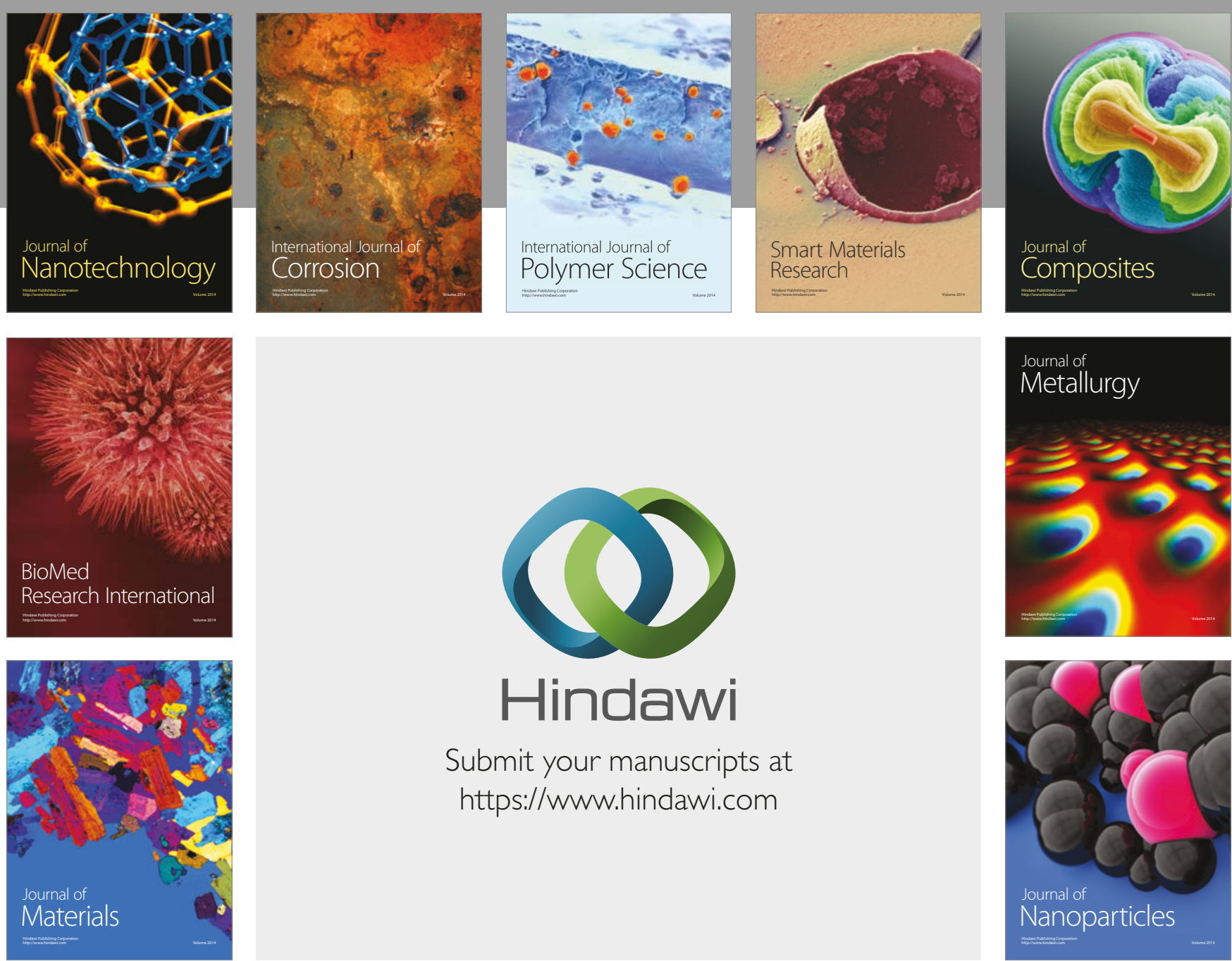

\section{Hindawi}

Submit your manuscripts at

https://www.hindawi.com
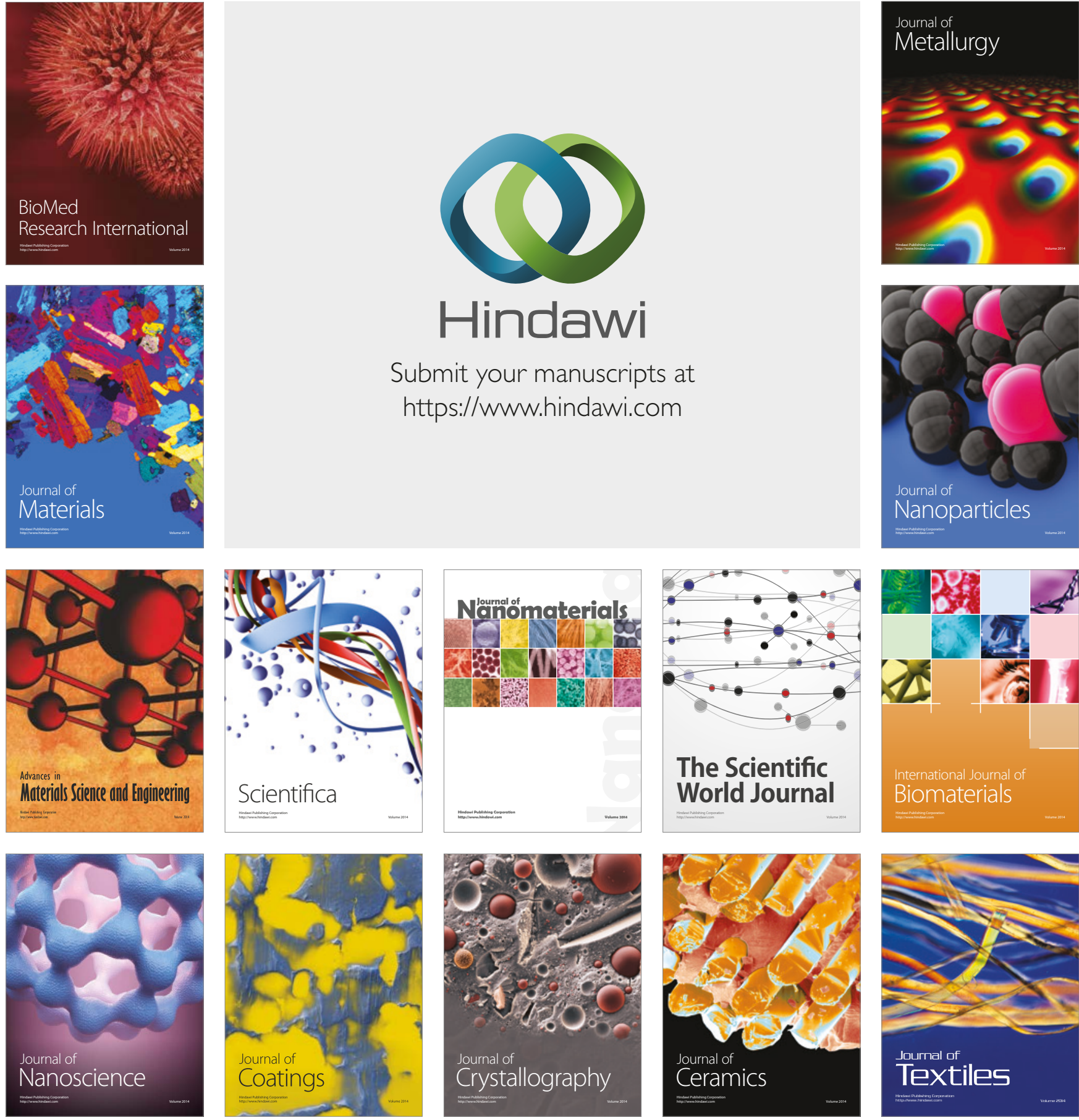

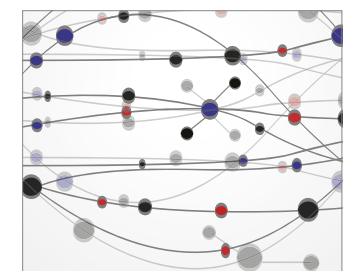

The Scientific World Journal
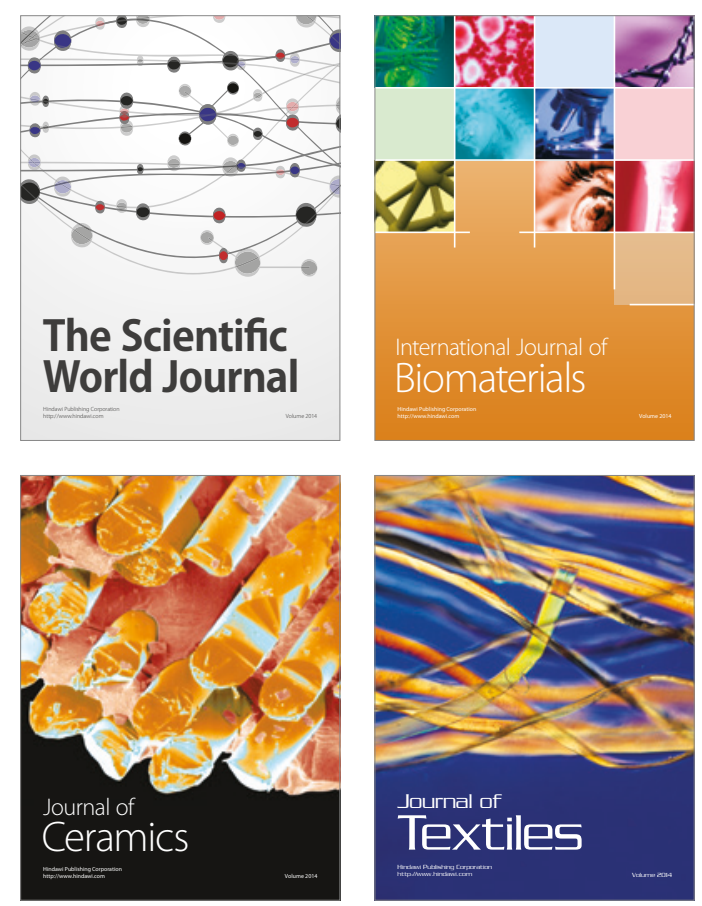